Pure and Applied Mathematics Quarterly

Volume 6, Number 3

(Special Issue: In honor of

Joseph J. Kohn, Part 1 of 2$)$

$663-675,2010$

\title{
Gevrey Hypoellipticity for Non-subelliptic Operators
}

\author{
Antonio Bove and David S. Tartakoff \\ Dedicated to Professor J.J.Kohn on the occasion of his 75th birthday
}

Abstract: In J. J. Kohn's recent paper [5] the operator

$$
E_{m, k}=L_{m} \overline{L_{m}}+\overline{L_{m}}|z|^{2 k} L_{m}, \quad L=\frac{\partial}{\partial z}-i \bar{z}|z|^{2(m-1)} \frac{\partial}{\partial t}
$$

was introduced and shown to be hypoelliptic, yet to lose $\frac{k-1}{m}$ derivatives in $L^{2}$ Sobolev norms. Christ [3] showed that the addition of one more variable destroyed hypoellipticity altogether. Here we show that this operator with an Oleinik-type singularity $E_{m, k}+|z|^{2(p-1)} \frac{\partial^{2}}{\partial s^{2}}$ is Gevrey hypoelliptic $G^{s}$ for any $s \geq \frac{2 m}{p-k},(2 m>p>k)$. A related result is that for the 'real' version, with $X=\frac{\partial}{\partial x}-i x^{q-1} \frac{\partial}{\partial t}$,

$$
R_{q, k}+x^{2(p-1)} \frac{\partial^{2}}{\partial s^{2}}=\bar{X}^{*} \bar{X}+\left(x^{k} X\right)^{*}\left(x^{k} X\right)+x^{2(p-1)} \frac{\partial^{2}}{\partial s^{2}}
$$

is Gevrey hypoelliptic $G^{s}$ for any $s \geq \frac{q}{p-k},(q>p>k)$, although the method of proof is different, and that the result is sharp.

The situation is reminiscent of the Baouendi-Goulaouic example, in which adding a new variable to a Grushin type analytic hypoelliptic operator destroys the analyticity and drops the regularity to $G^{2}$ yet prefacing that new second derivative by a power of the first variable to obtain an Oleinik-type operator improves the Gevrey index substantially (cf. [1]).

Keywords: Hypoellipticity; Gevrey regularity; Sums of squares of complex vector fields.

Received December 14, 2007.

[2000] Mathematics Subject Classification. Primary:35H10, 35H20; Secondary: 35A27, 35A30. 


\section{Introduction And StATEMENT of THEOREMS}

In sharp contrast to the situation with sums of squares of real vector fields whose iterated brackets span the full tangent space at each point and which consequently are subelliptic and both $C^{\infty}$ and Gevrey hypoelliptic, the operator introduced by J.J. Kohn:

$$
E_{k}=L \bar{L}+\bar{L}|z|^{2 k} L, \quad L=\frac{\partial}{\partial z}-i \bar{z} \frac{\partial}{\partial t}
$$

surprised analysts and geometers alike. This operator may be viewed as satisfying the bracket condition at each point but is far from being subelliptic, yet is hypoelliptic with loss of $k-1$ derivatives in $L^{2}$ Sobolev norms. In an Appendix to Kohn's paper, Derridj and the second author showed that $E_{k}$ was even analytic hypoelliptic and gave an alternative proof of Kohn's result. Later, in [9], the same result was proved for the more degenerate operator

$$
E_{m, k}=L_{m} \overline{L_{m}}+\overline{L_{m}}|z|^{2 k} L_{m}, \quad L_{m}=\frac{\partial}{\partial z}-i \bar{z}|z|^{2(m-1)} \frac{\partial}{\partial t}
$$

and further generalized were provided in [2], where the precise loss, namely $\frac{k-1}{m}$ for $E_{m, k}$, was established for still more general operators, in higher dimensions, although these operators seemed to require some radial symmetry in $|z|$.

Christ observed that the addition of a single new variable changed the situation dramatically, showing that neither $E_{k}+\frac{\partial^{2}}{\partial s^{2}}$ nor $R_{k}+\frac{\partial^{2}}{\partial s^{2}}$, is hypoelliptic, where

$$
R_{k}=\bar{X}^{*} \bar{X}+\left(x^{k} X\right)^{*}\left(x^{k} X\right), \quad X=\frac{\partial}{\partial x}-i x \frac{\partial}{\partial t} .
$$

Inspired by the work of Oleinik and that of Baouendi and Goulaouic, already generalized in [1], we study here the Gevrey hypoellipticity of the operators

$$
E_{m, k, p}=E_{m, k}+|z|^{2(p-1)} \frac{\partial^{2}}{\partial s^{2}}
$$

and, with $X_{q}=\frac{\partial}{\partial x}-i x^{q-1} \frac{\partial}{\partial t}$,

$$
R_{q, k, p}={\overline{X_{q}}}^{*} \overline{X_{q}}+\left(x^{k} X_{q}\right)^{*}\left(x^{k} X_{q}\right)+x^{2(p-1)} \frac{\partial^{2}}{\partial s^{2}},
$$

where $q$ is a positive even integer and $k \geq 0, p \geq 1$ are integers. We point out that if $q$ is odd then the field $\overline{X_{q}}$ is subelliptic because of Theorem 27.1.11 in [4], and that the operator $R_{q, k, p}$ behaves like the Oleinik operator no matter what 
the value of $k$ is (see e.g. [1] for the definition and Gevrey hypoellipticity results for the Oleinik operator.)

Our main result may be stated

Theorem 1. For $2 m>p>k$ (resp. $q>p>k$ ), the operator $E_{m, k, p}$ (resp. $R_{q, k, p}$ ) is Gevrey-s hypoelliptic for any $s \geq \frac{2 m}{p-k}$ (resp. $\left.s \geq \frac{q}{p-k}\right)$.

We would also like to emphasize that the Poisson-Treves stratification associated to $E_{m, k}$ or $R_{q, k}$ is symplectic, while the stratification for $E_{m, k, p}$ or $R_{q, k, p}$ is not.

\section{The a priori ESTIMATES}

We prefer to work with estimates in the inner product formulation, which are simple to derive, though require some additional work to yield regularity. (By contrast, Kohn's original paper used estimates in norms with explicit localizing functions, estimates which are far from simple to derive, but which have the advantage of yielding hypoellipticity at once.)

The estimate for $E_{m, k}$ for $v \in C_{0}^{\infty}\left(\mathbb{R}^{3}\right)$ is:

$$
\left\|\overline{L_{m}} v\right\|_{0}^{2}+\left\|\bar{z}^{k} L_{m} v\right\|_{0}^{2}+\|v\|_{-\frac{k-1}{2 m}(t)}^{2} \lesssim\left|\left(E_{m, k} v, v\right)_{0}\right|
$$

and hence, for $v \in C_{0}^{\infty}\left(\mathbb{R}^{4}\right)$,

$$
\left\|\overline{L_{m}} v\right\|_{0}^{2}+\left\|\bar{z}^{k} L_{m} v\right\|_{0}^{2}+\left\|z^{p-1} D_{s} v\right\|_{0}^{2}+\|v\|_{-\frac{k-1}{2 m}(t)}^{2} \lesssim\left|\left(E_{m, k, p} v, v\right)_{0}\right| .
$$

Remark 1. The negative norm need only be in the variable $t$, and $L^{2}$ in the other variables, as the derivation below will show. We note that $D_{t}$ commutes with every operator in this paper (and in Kohn's) which simplifies our computations.

Remark 2. We actually have a stronger estimate when $p=1$, i.e., the situation considered by Christ, since for small support (in s) the $L^{2}$ norm is bounded by a small multiple of the one norm in $s$, whence also

$$
\left\|\overline{L_{m}} v\right\|_{0}^{2}+\left\|\bar{z}^{k} L_{m} v\right\|_{0}^{2}+\left\|D_{s} v\right\|_{0}^{2}+\|v\|_{0}^{2} \lesssim\left|\left(E_{m, k, 1} v, v\right)_{0}\right|, \quad v \in C_{0}^{\infty}\left(\mathbb{R}^{4}\right),
$$

an estimate which appears to be substantially stronger, but is sufficiently far from the subelliptic case that hypoellipticity still fails. 
Analogously, for the 'real' case, the estimate for $R_{q, k}$ is:

$$
\left\|\overline{X_{q}} v\right\|_{0}^{2}+\left\|x^{k} X_{q} v\right\|_{0}^{2}+\|v\|_{-\frac{k-1}{q}(t)}^{2} \lesssim\left|\left(R_{q, k} v, v\right)_{0}\right|, \quad v \in C_{0}^{\infty}\left(\mathbb{R}^{3}\right),
$$

and hence, for $v \in C_{0}^{\infty}\left(\mathbb{R}^{4}\right)$,

$$
\left\|\overline{X_{q}} v\right\|_{0}^{2}+\left\|x^{k} X_{q} v\right\|_{0}^{2}+\left\|x^{p-1} D_{s} v\right\|_{0}^{2}+\|v\|_{-\frac{k-1}{q}(t)}^{2} \lesssim\left|\left(R_{q, k, p} v, v\right)_{0}\right| .
$$

Remark 3. All of our operators will have symplectic characteristic varieties for $z \neq 0$ (respectively $x \neq 0$ ) and thus localizing functions need only depend on the other variables in view of known results by Tartakoff, Treves and others.

Remark 4. For local real analyticity results, very careful localization of high powers of $\partial / \partial t$ has been required, namely

$$
\left(T^{r}\right)_{\varphi}=\varphi T^{r}+\sum W(\varphi) \tilde{W} T^{r-1}+\sum \frac{W^{2}(\varphi)}{2 !} \tilde{W}^{2} T^{r-2}+\ldots
$$

For Gevrey results this is not necessary, and for the local real analyticity of $R_{q, k}$ above it appears not even to be possible to construct $\left(T^{r}\right)_{\varphi}$ directly. We are fortunate to be able to complexify from $x$ to $z$, however, extend all solutions constant in $\Im z$, use the analytic result in the complex case, and then restrict back.

\section{Derivation of the estimate in the 'Real' Case}

For simplicity, we write the operator as

$$
R_{q, k, p}=X_{1}^{*} X_{1}+X_{2}^{*} X_{2}+x^{2(p-1)} \frac{\partial^{2}}{\partial s^{2}}=\sum_{j=1}^{3} W_{j}^{*} W_{j}
$$

with

$$
W_{1}=X_{1}=\frac{\partial}{\partial x}-i x^{q-1} \frac{\partial}{\partial t}, \quad W_{2}=X_{2}=x^{k} \overline{X_{1}}, \quad W_{3}=x^{p-1} \frac{\partial}{\partial s},
$$

and denote by $W_{4}$ the pseudo-differential power of the Laplacian in $t$

$$
W_{4}=\Lambda_{t}^{-\frac{k-1}{q}} .
$$

The a priori estimate to be established (always modulo a term of arbitrary negative order on the right hand side) is thus

$$
\sum_{j}^{3}\left\|W_{j} v\right\|_{0}^{2}+\|v\|_{-\frac{k-1}{q}(t)}^{2} \lesssim\left|\left(R_{q, k, p} v, v\right)_{0}\right|, \quad v \in C_{0}^{\infty} .
$$


To find the value of $\nu \leq 0$ (in $t$ ) for which

$$
\sum_{1}^{3}\left\|W_{j} v\right\|_{0}^{2}+\|v\|_{\nu}^{2} \lesssim\left|\left(R_{q, k, p} v, v\right)_{0}\right|, \quad v \in C_{0}^{\infty},
$$

we write $\|v\|_{\nu}^{2}=\left|\left(\left[\overline{X_{1}}, x\right] v, v\right)_{\nu}\right|$, so that, for $x$ small, since $\nu \leq 0$,

$$
\begin{aligned}
\|v\|_{\nu}^{2} \leq\left|\left(x \overline{X_{1}} v, v\right)_{\nu}\right|+\text { s.c. }\|v\|_{\nu}^{2}+\text { l.c. } \| & X_{1} v \|_{0}^{2} \\
& \leq \text { l.c. }\left\|x \overline{X_{1}} v\right\|_{\nu}^{2}+\text { s.c. }\|v\|_{\nu}^{2}+\text { l.c. }\left\|X_{1} v\right\|_{0}^{2}
\end{aligned}
$$

Here l.c. denotes a "large" positive constant and s.c. an "arbitrarily small" positive constant. In the first term on the right we raise and lower powers of $x \Lambda_{t}^{\frac{1}{q}}$, then integrate by parts, and finally commute $X_{1}$ and $\overline{X_{1}}$ :

$$
\begin{aligned}
\text { l.c. }\left\|x \overline{X_{1}} v\right\|_{\nu}^{2} & \leq \text { s.c. }\left\|\overline{X_{1}} v\right\|_{\nu-\frac{1}{q}}^{2}+\text { l.c. }\left\|x^{k} \overline{X_{1}} v\right\|_{\nu+\frac{k-1}{q}}^{2} \\
& \leq \text { s.c. }\left\|X_{1} v\right\|_{\nu-\frac{1}{q}}^{2}+\text { s.c. }\left\||x|^{\frac{q-2}{2}} \Lambda_{t}^{\frac{1}{2}-\frac{1}{q}} v\right\|_{\nu}^{2}+\text { l.c. }\left\|x^{k} \overline{X_{1}} v\right\|_{0}^{2} \\
& \leq \text { l.c. }\left\{\left\|X_{1} v\right\|_{0}^{2}+\left\|x^{k} \overline{X_{1}} v\right\|_{0}^{2}\right\}+\text { s.c. }\left\||x|^{\frac{q-2}{2}} \Lambda_{t}^{\frac{1}{2}-\frac{1}{q}} v\right\|_{\nu}^{2}
\end{aligned}
$$

if $\nu=-\frac{k-1}{q}$; raising and lowering powers of $x \Lambda_{t}^{\frac{1}{q}}$ once more,

$$
\begin{gathered}
\left\||x|^{\frac{q-2}{2}} \Lambda_{t}^{\left(\frac{1}{q}\right)\left(\frac{q-2}{2}\right)} v\right\|_{\nu}^{2} \leq\|v\|_{\nu}^{2}+\left\||x|^{\frac{q}{2}-1+k} \Lambda^{\frac{1}{2}+\frac{k-1}{q}} v\right\|_{\nu}^{2} \\
=\|v\|_{\nu}^{2}+\left\||x|^{\frac{q}{2}-1+k} \Lambda^{\frac{1}{2}} v\right\|_{0}^{2}=\|v\|_{\nu}^{2}+\left|\left(\left[X_{1}, x^{k} \overline{X_{1}}\right] v, x^{k} v\right)_{0}\right| \\
\leq\|v\|_{\nu}^{2}+\left\|X_{1} v\right\|_{0}^{2}+\left\|x^{k} \overline{X_{1}} v\right\|_{0}^{2}+\left\|x^{k-1} v\right\|_{0}^{2},
\end{gathered}
$$

since the 'Levi form' is bounded by the sum of the operators modulo this last error. However,

$$
\left\|x^{k-1} v\right\|_{0}^{2} \sim\left|\left(\left[X_{1}, x^{2 k-1}\right] v, v\right)_{0}\right| \leq\left|\left(x^{k-1} v, x^{k} \overline{X_{1}} v\right)_{0}\right|+\left(X_{1} v, x^{2 k-1} v\right)_{0} \mid
$$

which shows that this error is harmless.

Thus, since $W_{2}=x^{k} \overline{X_{1}}$, and including $W_{3}=x^{p-1} \frac{\partial}{\partial s}$ is trivial,

$$
\sum_{1}^{3}\left\|W_{j} v\right\|_{0}^{2}+\|v\|_{-\frac{k-1}{q}(t)}^{2} \lesssim\left|\left(R_{q, k, p} v, v\right)_{0}\right|,
$$

for $v$ of small support. The same derivation, distinguishing when necessary between $z$ and $\bar{z}$, applies to the Oleinik variant of Kohn's operator, $E_{m, k, p}$. 


\section{4. $C^{\infty}$ Hypoellipticity}

Microlocally, our operators are elliptic for $\xi \neq 0$ (or $\zeta \neq 0$ ), hence even analytic hypoelliptic there. Hence the challenge is to bound growth of derivatives in $s$ and $t$ suitably. And since all coefficients are independent of $(s, t)$, in either case $P=E_{m, k, p}$ or $P=R_{q, k, p}$ we may multiply the equation $P u=f$ through by a pseudodifferential operator $\Psi=\Psi_{N}$ with smooth symbol

$$
\Psi_{N}(\sigma, \tau) \equiv 1,|\sigma|+|\tau| \leq N, \quad \Psi_{N}(\sigma, \tau) \equiv 0,|\sigma|+|\tau| \geq 2 N
$$

where $\sigma$ is dual to $s$ and $\tau$ to $t$. (Derivatives of $\Psi$ will never enter, since $[\Psi, P]=0$.) Then for our solution $u$, we have $\Psi_{N}\left(\frac{\partial}{\partial s}, \frac{\partial}{\partial t}\right) u \in C^{\infty}$ and, making sure that our estimates are uniform in $N$, in the end letting $N \rightarrow \infty$ we may obtain corresponding estimates on the solution $u$ itself and the finiteness of the norms involved. Thus we will work as though the solution $u$ were already smooth.

\section{Gevrey Hypoellipticity, Real Case}

Since $q>p$, the critical direction is $T=\partial / \partial t$. And while strictly speaking we should introduce localization in all variables, any localizing function in $x$ will be merely passively dragged along - when it is differentiated we land in a region bounded away from $x=0$, and the result is known there, so we will suppress localization in $x$. Further, while $T$ will occur with non-integral powers, we should strictly speaking localize on both the left and the right of $T$, with the function on the right identically equal to one near that on the left. But again, when this function on the right is differentiated we wind up with a pseudo-differential operator with symbol identically equal to zero, hence sufficiently smoothing if the localizing functions are in the Enrenpreis class. Thus we will suppress this second localization to the right of $T$ as well. In fact, derivatives in $S=\frac{\partial}{\partial s}$ will grow analytically, since the exponent $p-1$ satisfies $p<q$ (resp. $p<2 m$.) and all errors which arise will still contain the coefficient $x^{p-1}$ which may be combined with one of the powers of $S$ to make a maximally bounded vector field, denoted $W_{3}$ in both cases.

Given a solution $u$, then, assumed to be smooth, to $R_{q, k, p} u=f \in G^{\sigma}$, we will set $v=\varphi(s, t) T^{r} u$ in the a priori estimate and commute $\varphi(s, t) T^{r}$ past $R_{q, k, p}$ with acceptable errors. 
In using (R) with $v=\varphi(s, t) T^{r} u$, the critical brackets which enter at once are

$$
\left\|\left[W_{j}, \varphi T^{r}\right] u\right\|_{0}^{2}, \quad j=1,2,3 .
$$

In more detail,

$$
\left\|\left[W_{j}, \varphi T^{r}\right] u\right\|_{0}^{2}=\left\|x^{\ell_{j}} \varphi^{\prime} T^{r} u\right\|_{0}^{2},
$$

with $\ell_{1}=q-1, \ell_{2}=q+k-1, \ell_{3}=p-1$, and $\varphi^{\prime}$ denoting a first derivative of $\varphi$ in $t(j=1,2)$ or in $s(j=3)$.

To treat these, we use the same techniques as above, since for $q, p>1$, we may always raise and lower powers of $\left(x \Lambda_{t}\right)$. When $p=1$ (Christ's example) there is no $x$ in the third bracket and hence we cannot apply these techniques.

Now, since $\ell_{3}=p-1$ is the smallest of the $\ell_{j}$ and hence the hardest to treat, and for any $\delta>\frac{k-1}{q}$,

$$
\begin{aligned}
\left\|\left[W_{j}, \varphi T^{r}\right] u\right\|_{0}^{2}= & \left\|x^{\ell_{j}} \varphi^{\prime} T^{r+\frac{k-1}{q}} u\right\|_{-\frac{k-1}{q}}^{2} \\
\lesssim & \left\|\varphi^{\prime} T^{r+\frac{k-1}{q}-\delta} u\right\|_{-\frac{k-1}{q}}^{2} \\
& +\left\|x^{q+k-1} \varphi^{\prime} T^{r+\frac{k-1}{q}+\frac{\delta}{p-1}(q+k-1-(p-1))} u\right\|_{-\frac{k-1}{q}}^{2}
\end{aligned}
$$

or, for the complex case below, with $q=2 m$ but $\ell_{3}$ still the smallest of the $\ell_{j}$, we may raise the powers of $x$ (now $|z|$ ) less, still writing $W_{j}^{c}$ now for $\overline{L_{m}}, \bar{z}^{k} L_{m}$, or $z^{p-1} \frac{\partial}{\partial s}$,

$$
\begin{aligned}
\left\|\left[W_{j}^{c}, \varphi T^{r}\right] u\right\|_{0}^{2} \leq & \left\|z^{\ell_{j}} \varphi^{\prime} T^{r+\frac{k-1}{q}} u\right\|_{-\frac{k-1}{q}}^{2} \\
\lesssim & \left\|\varphi^{\prime} T^{r+\frac{k-1}{q}-\delta} u\right\|_{-\frac{k-1}{q}}^{2} \\
& +\left\||z|^{\frac{q-2}{2}+k} T^{\frac{1}{2}} \varphi^{\prime} T^{r+\frac{k-1}{q}+\frac{\delta}{p-1}\left(\frac{q-2}{2}+k-(p-1)\right)-\frac{1}{2}} u\right\|_{-\frac{k-1}{q}}^{2}
\end{aligned}
$$

For the real case, $x^{k+q-1} T$ can be written as a linear combination of $X_{1}$ and $X_{2}=x^{k} \overline{X_{1}}$ since $X_{1}=\frac{\partial}{\partial x}-i x^{q-1} \frac{\partial}{\partial t}$. This makes the last term in the estimate for brackets with $W_{j}$ just above essentially

$$
\left\|W \varphi^{\prime} T^{r+\frac{\delta}{p-1}(q+k-1-(p-1))-1} u\right\|_{0}^{2},
$$

which gives a gain of $1-\frac{\delta}{p-1}(q+k-1-(p-1))$ for one derivative on $\varphi$, while the first term gives a gain of $\delta-\frac{k-1}{q}$. These lead quickly to the Gevrey index. Choosing $\delta$ suitably will minimize the Gevrey index. 


\section{Gevrey Hypoellipticity, Complex Case}

For the complex case of

$$
E_{m, k, p}=E_{m, k}+|z|^{2(p-1)} \frac{\partial^{2}}{\partial s^{2}}=\sum_{j} W_{j}^{c *} W_{j}^{c},
$$

we use the estimate for brackets with $W_{j}^{c}$ just above, since now the operators $L$ and $\bar{L}$ are not sufficiently similar to permit the $T$ term to be written as their linear combination $\left(\frac{\partial}{\partial z}\right.$ and $\frac{\partial}{\partial \bar{z}}$ will not cancel as in the real case) so we have to consider the second term in (5.2) as a Levi form, and bound it by the vector fields with additional loss. That is, this term, in complex form, is estimated by

$$
\begin{gathered}
\left\||z|^{\frac{q-2}{2}+k} T^{\frac{1}{2}} \varphi^{\prime} T^{r+\frac{k-1}{q}+\frac{\delta}{p-1}\left(\frac{q-2}{2}+k-(p-1)\right)-\frac{1}{2}} u\right\|_{-\frac{k-1}{q}}^{2} \\
\lesssim \sum_{j}\left\|W_{j}^{c} \varphi^{\prime} T^{r+\frac{\delta}{p-1}\left(\frac{q-2}{2}+k-(p-1)\right)-\frac{1}{2}} u\right\|_{0}^{2}
\end{gathered}
$$

leading to a gain of $\frac{1}{2}-\frac{\delta}{p-1}\left(\frac{q-2}{2}+k-(p-1)\right)$ for each derivative on $\varphi$. Again, the Gevrey index is rapidly computed.

\section{And the Real Answer Is ...}

For the 'real' case, the condition was - with $\delta>\frac{k-1}{q}$, Gevrey hypoellipticity held in $G^{\sigma}$ with

$$
\sigma=\min _{\delta>\frac{k-1}{q}} \max \left(\frac{1}{\delta-\frac{k-1}{q}}, \frac{1}{1-\frac{\delta}{p-1}(q+k-p)}\right)
$$

and this occurs when the denominators are equal, namely for $\delta=\frac{p-1}{q}$, yielding

$$
\min _{\delta>\frac{k-1}{2 m}} \max \left(\frac{1}{\delta-\frac{k-1}{q}}, \frac{1}{1-\frac{\delta}{p-1}(q+k-p)}\right)=\frac{q}{p-k},
$$

$(q>p>k)$.

\section{And the Complex Answer Is . . .}

For the 'complex' case, the condition is - with $\delta>\frac{k-1}{2 m}$, Gevrey hypoellipticity held in $G^{\sigma}$ with

$$
\sigma=\min _{\delta>0} \max \left(\frac{1}{\delta-\frac{k-1}{2 m}}, \frac{1}{\frac{1}{2}-\frac{\delta}{p-1}\left(\frac{2 m}{2}+k-p\right)}\right)
$$


and this occurs when the denominators are equal, namely for $\delta=\frac{p-1}{2 m}$, yielding

$$
\min _{\delta>0} \max \left(\frac{1}{\delta-\frac{k-1}{2 m}}, \frac{1}{\frac{1}{2}-\frac{\delta}{p-1}\left(\frac{2 m}{2}+k-p\right)}\right)=\frac{2 m}{p-k},
$$

$(2 m>p>k)$.

\section{Sharpness}

We want to show that the Gevrey regularity for $R_{q, k, p}$ given above is sharp and cannot be improved. As a preliminary fact, we need to show that there is a suitable value of the parameter $\lambda$ such that the ordinary differential equation

$$
\hat{R}_{q, k}(\tau) u+\lambda \tau^{-2 \frac{k}{q}} x^{2(p-1)} u=0
$$

has a non-zero solution in $\mathscr{S}(\mathbb{R})$. Here $\tau$ is a large positive parameter and $\hat{R}_{q, k}(\tau)$ denotes the operator

$$
\hat{R}_{q, k}(\tau)=X^{*} X+\tau^{-2 \frac{k}{q}}\left(x^{k} X^{*}\right)^{*} x^{k} X^{*},
$$

where

$$
X=D_{x}-i x^{q-1}, \quad D_{x}=i^{-1} \partial_{x}
$$

so that $X^{*}=D_{x}+i x^{q-1}$.

This is accomplished in two steps:

- Show that for $u \in \mathscr{S}(\mathbb{R})$ we have the following estimate

$$
\left\langle\hat{R}_{q, k} u, u\right\rangle \geq c \tau^{-2 \frac{k}{q}}\|u\|^{2},
$$

with a positive constant $c$ independent of $\tau$ and $u$.

- Argue as in Lemma 1 of [7] to reach the conclusion.

We shall actually need an estimate slightly different from (9.2) but which will follow from it.

Lemma 9.1. Let $q$ and $k$ be positive integers. Then for every $u \in \mathscr{S}(\mathbb{R})$ we have

$$
\left\langle\hat{R}_{q, k} u, u\right\rangle \geq c \tau^{-2 \frac{k}{q}}\|u\|^{2},
$$

with a positive constant $c$ independent of $\tau$ and $u$. Here $\|\cdot\|$ and $\langle\cdot, \cdot\rangle$ denote the $L^{2}$ norm and scalar product in $\mathbb{R}$. 
Proof. We start off by noting that

$$
\tau^{-2 \frac{k}{q}}\|u\|^{2}=\tau^{-2 \frac{k}{q}}\left\langle\left[X^{*}, x\right] u, u\right\rangle=\tau^{-2 \frac{k}{q}}\left\{\langle x u, X u\rangle-\left\langle x X^{*} u, u\right\rangle\right\} .
$$

We estimate each of the two terms above. We have, since

$$
|x| \leq \varepsilon+C_{\varepsilon, m}|x|^{m}
$$

for any $\varepsilon$ and $m$,

$$
\begin{aligned}
\left|\left\langle x X^{*} u, u\right\rangle\right| & \leq \text { s.c. }\|u\|^{2}+\text { l.c. }\left\|x X^{*} u\right\|^{2} \\
& \leq \text { s.c. }\|u\|^{2}+\text { s.c. }\left\|X^{*} u\right\|^{2}+\text { l.c. }\left\|x^{k} X^{*} u\right\|^{2} .
\end{aligned}
$$

The first term in the second line above can be absorbed on the left. The last term is good when combined with the power of $\tau$ in front of it. We have to examine the second term.

For the second term we choose a compact set $K=\left[-\frac{1}{2}, \frac{1}{2}\right]$ and split $\left\|X^{*} u\right\|=$ $\left\|X^{*} u\right\|_{K}+\left\|X^{*} u\right\|_{K^{c}}$. We have

$$
\begin{aligned}
\left\|X^{*} u\right\|_{K} \leq\|X u\|_{K}+ & \left\|\left(X-X^{*}\right) u\right\|_{K} \leq\|X u\|_{\mathbb{R}}+2\left\|x^{q-1} u\right\|_{K} \\
& \leq\|X u\|_{\mathbb{R}}+2\|u\|_{\mathbb{R}}
\end{aligned}
$$

while for the region $K^{c}$ we have

$$
\left\|X^{*} u\right\|_{K^{c}} \leq 2^{k}\left\|x^{k} X^{*} u\right\|_{K^{c}} \leq 2^{k}\left\|x^{k} X^{*} u\right\|_{\mathbb{R}}
$$

so that together,

$$
\left\|X^{*} u\right\| \leq\|X u\|_{\mathbb{R}}+2\|u\|_{\mathbb{R}}+C\left\|x^{k} X^{*} u\right\|_{\mathbb{R}}
$$

For the other term, $|\langle x u, X u\rangle|$, we write

$$
|\langle x u, X u\rangle| \leq \text { l.c. }\|X u\|^{2}+\text { s.c. }\|x u\|^{2} \leq \text { l.c. }\|X u\|^{2}+\text { s.c. }\|u\|^{2}+\text { s.c. }\left\|x^{q-1} u\right\|^{2}
$$

as before and write

$$
\left\|x^{q-1} u\right\|^{2}=\frac{1}{2}\left\|\left(X-X^{*}\right) u\right\|^{2} \leq\left\{\|X u\|^{2}+\left\|X^{*} u\right\|^{2}\right\}
$$

and we have treated the last term just above. Note that, unlike [9] and [2], where we were able to take $u$ to have small support, we can not take $\|x u\|$ to be estimated by a small multiple of $\|u\|$.

This completes the proof of the lemma.

The next lemma has the estimate we need. 
Lemma 9.2. Let $p, q$ and $k$ be positive integers such that $q>p>k$. Then for every $u \in \mathscr{S}(\mathbb{R})$ we have

$$
\left\langle\hat{R}_{q, k} u, u\right\rangle \geq c \tau^{-2 \frac{k}{q}}\left\|x^{p-1} u\right\|^{2}
$$

with a positive constant $c$ independent of $\tau$ and $u$.

Proof. It suffices to observe that since $|x|^{p-1} \lesssim 1+|x|^{q-1}$, it suffices to estimate both $\|u\|$ and $\left\|x^{q-1} u\right\|$ both of which we have done above. This ends the proof of the lemma.

Lemma 9.3. Under the hypotheses in Lemma 9.2, there exists a positive constant $\lambda$ such that the ordinary differential equation

$$
\hat{R}_{q, k}(\tau) u+\lambda \tau^{-2 \frac{k}{q}} x^{2(p-1)} u=0
$$

has a non zero solution in the domain in $L^{2}$ of $\hat{R}_{q, k}(\tau)$.

Proof. This is the same argument as in Lemma 1 of [7], once we point out that the $L^{2}$ domain of the operator $\hat{R}_{q, k}(\tau), \tau>0$, is compactly embedded in $L^{2}$.

The argument of Lemma 1 in [7] is pursued by showing that a minimizing sequence for the ratio

$$
\inf _{u \in \mathscr{S}(\mathbb{R})} \frac{\left\langle\hat{R}_{q, k} u, u\right\rangle}{\tau^{-2 \frac{k}{q}}\left\langle x^{2(p-1)} u, u\right\rangle}
$$

exists and converges with respect to the norm $\|u\|^{2}=\left\langle\hat{R}_{q, k} u, u\right\rangle$. Thanks to Lemma 9.2 we see that the infimum does not depend on $\tau$. We refer the reader to [7] for the details.

Let us denote by $v_{\tau}(x)$ the solution of (9.4) determined by Lemma 9.3. We point out explicitly that $v_{\tau}$ satisfies an estimate of the form

$$
\left|v_{\tau}(x)\right| \leq C e^{-\frac{|x| q}{q}}\left(1+\mathscr{O}\left(\tau^{-\frac{k}{q}}\right)\right),
$$

when $|x| \rightarrow+\infty$ (see e.g. [8]); here $\mathscr{O}\left(\tau^{-\alpha}\right)$ denotes a quantity uniformly bounded w.r.t. the $x$-variable for $|x| \rightarrow+\infty$.

Define

$$
u(x, s, t)=\int_{0}^{+\infty} \exp \left(i \rho t+i \lambda^{\frac{1}{2}} \rho^{-\frac{k}{q}+\frac{p}{q}} s-\rho^{\theta}\right) v_{\rho}\left(\rho^{\frac{1}{q}} x\right) d \rho,
$$


where

$$
\theta>\frac{p-k}{q}
$$

It is easily checked that the integral in (9.5) is absolutely convergent and that $u$ is a null solution of the equation

$$
R_{q, k, p}\left(x, D_{x}, D_{s}, D_{t}\right) u=0 .
$$

It is now straightforward to compute

$$
\left|\partial_{t}^{j} u(0)\right| \sim C \int_{0}^{+\infty} e^{-\rho^{\theta}} \rho^{j} d \rho \sim C^{j} j !^{\frac{1}{\theta}},
$$

which proves

Theorem 2. The operator $R_{q, k, p}\left(x, D_{x}, D_{s}, D_{t}\right)$ is $G^{\sigma}$ hypoelliptic, for $\sigma \geq \frac{q}{p-k}$ and not for any $\sigma<\frac{q}{p-k}$.

\section{REFERENCES}

[1] A. Bove, D. S. Tartakoff, Optimal non-isotropic Gevrey exponents for sums of squares of vector fields, Comm. Partial Differential Equations 22 (1997), no. 7-8, 1263-1282.

[2] A. Bove, M. Derridj, J. J. Kohn and D. S. Tartakoff, Sums of Squares of Complex Vector Fields and (Analytic-) Hypoellipticity, Math. Res. Lett. 13(2006), no. 5-6, 683-701.

[3] M. Christ, A remark on sums of squares of complex vector fields, arXiv:math.CV/0503506.

[4] L. Hörmander, The Analysis of Linear Partial Differential Operators, Springer Verlag, 1983-1985.

[5] J.J. Kohn, Hypoellipticity and loss of derivatives, Ann. of Math. (2) 162 (2005) 943-982.

[6] O. Oleĭnik, On the analyticity of solutions of partial differential equations and systems, Colloque International, CNRS sur les quations aux Drives Partielles Linaires (Univ. ParisSud, Orsay, 1972), pp. 272-285. Asterisque, 2 et 3. Soc. Math. France, Paris, 1973.

[7] O. A. Oleŭnik, E. V. Radkevič, The analyticity of the solutions of linear partial differential equations, (Russian) Mat. Sb. (N.S.) 90(132)(1973), 592-606.

[8] Y. SiBuYA, Global Theory of a Second Order Linear Ordinary Equation with a Polynomial Coefficient, North-Holland Mathematical Studies vol. 18, 1975.

[9] D. S. Tartakoff, Analyticity for singular sums of squares of degenerate vector fields, Proc. Amer. Math. Soc. 134 (2006), no. 11, 3343-3352.

Antonio Bove

Dipartimento di Matematica,

Università di Bologna,

Piazza di Porta San Donato 5,

Bologna ITALY 
E-mail: bove@bo.infn.it

David S. Tartakoff

Department of Mathematics

University of Illinois at Chicago,

m/c 249, 851 S. Morgan St.,

Chicago IL 60607, USA

E-mail: dst@uic.edu 